\title{
Fungal pellets as potential tools to control water pollution: Strategic approach for the pelletization and subsequent microcystin-LR uptake by Mucor hiemalis
}

\author{
Evelyn Balsano ${ }^{\mathrm{a}}$, Maranda Esterhuizen-Londt ${ }^{\mathrm{a}}$, Enamul Hoque ${ }^{\mathrm{c}}$, Stephan Pflugmacher ${ }^{\mathrm{a}, \mathrm{b}^{*}}$ \\ ${ }^{a}$ Chair of Ecotoxicological Impact Research and Ecotoxicology, Technische Universität Berlin, Ernst-Reuter-Platz 1, 10587 Berlin, Germany. \\ ${ }^{b}$ Center for Water Resource Cycle Research, Korea Institute of Science and Technology (KIST), 39-1 Hawarangno 14-gil 5, Seongbuk-gu, Seoul, 136-791, \\ Republic of Korea. \\ ${ }^{c}$ Helmholtz Zentrum München - German Research Center for Environmental Health, Institute of Groundwater Ecology, Ingolstädter Landstr. 1, 85764 \\ Neuherberg, Germany.
}

\section{ARTICLE INFO}

Article history:

Received on: 13/10/2015

Revised on: 21/12/2015

Accepted on: 23/02/2016

Available online: 26/08/2016

Key words:

Mucor hiemalis, pelletization, mycoremediation,

microcystin-LR, uptake.

\begin{abstract}
Microcystin-LR is one of the most prevalent and toxic secondary metabolites produced by cyanobacteria worldwide, causing global concerns because of its hazardousness to ecosystems and human health. Green Liver Systems ${ }^{\circledR}$ have been developed to purify contaminated water, however, system capacities need to be extended to allow season- and location independent applications. Therefore, mycoremediation using temperature resistant Mucor hiemalis in pellet morphology was considered. In submerged liquid cultures, fungal morphology is species specific and strongly depends on the cultivation environment. One main focus of the present study was the investigation of diverse factors influencing pelletization. Moreover, we translated the pellet product into an immediate application and studied its biosorption ability towards microcystin-LR. Our results showed that $\mathrm{pH}$ was a key factor stimulating pellet formation of $M$. hiemalis and that inoculum size played an essential role as well. Final pellet size was limited by the available space in the flask and is therefore directly related to inoculum size. Microcystin-LR was found to be taken up by pelletized M. hiemalis as quantified via LC-MS/MS measurements. Our results report for the first time optimized pelletization of $M$. hiemalis and cyanotoxin uptake by these fungal pellets in liquid cultures.
\end{abstract}

\section{INTRODUCTION}

In the last decades, concern regarding the negative effects of cyanobacterial toxins on aquatic ecosystems as well as human health has grown worldwide. Microcystins are heptapeptides, which attracted attention not only due to their high acute and chronic toxicities but also due to their global abundance. Microcystin-LR (MC-LR) in particular, is considered the most toxic candidate of this family [1] and is produced by cyanobacterial species belonging to the genera Anabaena, Microcystis, Nostoc and Anabaenopsis [2-5]. It is toxic to the liver, promoting liver tumors in humans [6-10], and showed to be

\section{* Corresponding Author}

Prof. Dr. Stephan Pflugmacher, Chair of Ecotoxicological Impact Research and Ecotoxicology, Technische Universität Berlin, ErnstReuter-Platz 1, 10587 Berlin, Germany. Tel.: +493031429023, Fax: +49 3031429022. Email: stephan.pflugmacher@tu-berlin.de. acutely toxic in mice [11]. In the aquatic environment, it can have significant negative effects on the survival of zooplankton including species of Daphnia [12]. Investigations of the effect in different early life stages of zebrafish (Danio rerio) showed uptake of the toxin resulting in growth reduction and malformations [13]. Phytotoxic effects were studied in various aquatic plants such as Lemna gibba $[14,15]$ and Ceratophyllum demersum [16]; a clear dose-dependent inhibition of macrophyte growth with exposure to low concentrations of MC-LR has been shown. Reduction in growth, rate of photosynthesis and changes in plant pigment composition were observed [17]. The stable cyclic structure of MCs makes conventional water treatment (such as chlorination, chlorine dioxide, and ozonation) and physicochemical techniques a challenge, as the removal capability remains limited [18]. More advanced methods (such as activated carbon in granular or powder form and membrane filtration) result in high costs and therefore, the investigation and development of more effective and low-cost water treatment technologies is of great importance in order to 
ensure the removal of cyanotoxins from aquatic ecosystems. Green Liver Systems ${ }^{\circledR}$ present a possible way for the purification of cyanotoxin-contaminated water bodies in a cost-effective, sustainable and environmentally friendly manner. The system uses the phytoremediation potential of aquatic submerged plants [19], i.e. the capability to take up contaminants from the water, biotransform them enzymatically and lastly, store them in cell wall fractions, the apoplast or in the vacuoles. Successful results in respect to cyanobacterial toxin removal were obtained in simulating Green Liver Systems ${ }^{\circledast}$ in the laboratory and as well in a small pilot plant in Hefei (Anhui Region, PR China) [20] and Itacuruba (Nordeste Region, Brazil) [19]. Despite the promising remediation capacities of Green Liver Systems ${ }^{\circledR}$, their application is limited to warm seasons and climates with temperature ranges suitable for plant growth, development and survival. Hence, there is a demanding need to search for alternative bioremediating agents that could be applied season-independently and worldwide for effective purification of cyanotoxin-contaminated water bodies. It is therefore of high scientific interest to investigate the applicability of further organisms as an alternative to aquatic macrophytes currently used in Green Liver Systems ${ }^{\circledR}$. Microorganisms that play a major role in decomposition and degradation in the ecosystem, based on natural functions are the fungi. Mycoremediation, as a process of using fungi to degrade contaminants in the environment, offers a wide application area and has raised international interest throughout the last decades. Via non-specific extracellular enzymes (peroxidase, manganese peroxidase, laccase), fungi are able to breakdown many persistent complex organic substances, such as lignin and cellulose [21, 22], and are therefore responsible for wood decay. This natural degradation ability of the non-specificity of the fungal enzyme machinery is used for industrial applications to degrade, mineralize and remove other natural or chemical organic pollutants as well [23]. Many white-rot fungi are known to degrade major environmental pollutants such as munitions waste, pesticides, organochlorines, polychlorinated biphenyls (PCBs), polycyclic aromatic hydrocarbons (PAHs), synthetic dyes, wood preservatives and synthetic polymers [24]. Phanerochaete chrysosporium is extensively used as a model for white-rot fungal biodegradation through the involvement of the lignin degrading system. Mineralization of chloraniline/lignin conjugates, free chloraniline and their produced metabolites has been successfully characterized $[25,26]$. The fungus proved to remediate atrazinecontaminated soils [27, 28], various PAHs [29], PCBs [30], TNT [31], pentachlorophenol [32], 2,4,5-trichlorophenol [33] and many more. A recent high-tech application is the use of $P$. chrysosporium as a tool in different fungal bioreactor systems for the bleaching in Kraft plant effluents [34], the removal of phenolic compounds from coking wastewater [35], or the decolorization of textile dyes [36, 37].

Overall, the use of fungi may be a promising alternative to plant organisms, however, similar drawbacks are encountered in regard to temperature limitation of white-rot fungi. $P$. chrysosporium for example, has a growth optimum of $30-39{ }^{\circ} \mathrm{C}$
[38], therefore, cultivation would lead to energy expenditure in biotechnological processes and would still not be the solution for a universal and season-independent bioremediating system. In order to overcome temperature limitations, the search for an appropriate fungal organism has to be extended. Instead of using fungi with peroxidase enzymes, one can focus on fungi with glutathioneStransferase activity. Mucor hiemalis is an aquatic fungus that is known to express high levels of extracellular glutathioneStransferases [39], which are a class of detoxification enzymes. Its degradation potential has been highlighted in regard to the herbicide isoproturon [40]. Moreover, it displays functional groups on the cell wall surface that enable absorption of heavy metals and is therefore used for the removal of nickel [41], chrome [42], mercury, etc. from ground- and surface water, purification plants, waste water and industrial water [43]. Most importantly, the fungus still sporulates even at ground water temperatures of $5{ }^{\circ} \mathrm{C}$ and is resistant to high hydrogen sulfide concentrations [39]. Our previous study showed its resistance to three globally occurring and structural diverse cyanobacterial toxins [44]. Growth and development were not affected in the presence of these toxins and uptake could be detected in the mycelium after exposure. Xenobiotic resistance and good biosorption ability are prerequisites for the use of the fungus as a bioremediating agent. These promising results sustain further research to answer the question: Is $M$. hiemalis a possible alternative for the remediation of cyanotoxins from contaminated waters? Hence, investigations on uptake in submerged liquid cultures have to be undertaken in preliminary laboratory experiments. As the present fundamental research will serve for the development of future fungal bioreactors, it is important to establish systems that are operating with the ideal morphological growth form. Concerning biotechnological applications, it may be useful to develop devices that work with pelletized fungal biomass. The filamentous mycelial growth causes problems in bioreactors as it could interfere with bioreactor components leading to decreased productivity, reduced growth and negatively affecting the potential for bioremediation [45]. Hence, using compact pelletized fungal biomass in bioreactor technology provides many advantages, including decreased broth viscosity, easier separation, improved aeration, stirring, heat transfer, and a larger surface area which reduces the mass transfer limitations and enhances uptake [46, 47]. Many filamentous fungi have the ability to grow in the form of small spherical pellets of intertwined hyphae, however, this morphology has been shown to be species specific and to strongly depend on cultural conditions. Pellet formation has been extensively studied for different filamentous fungi, and many approaches for the pellet formation of Rhizopus (R.) spp. [48-51] and different species of the genus Aspergillus (A.) [52-54] have been found. The factors affecting pellet formation are not only genus but also species specific. To the authors' knowledge, pellet formation of only one Mucor species has yet been reported [55]. The aim of this work was therefore to investigate the optimal condition for the pelletization of $M$. hiemalis, as to date no information exists on pellet formation of this species and 
pelletized fungal biomass offers many advantages in biotechnological applications. Investigations of various factors that have been reported to influence fungal morphology, such as temperature, medium composition, $\mathrm{pH}$, inoculum size, additives, agitation rate, volume, or flask shape are presented. Finally, we demonstrate the ability of $M$. hiemalis to incorporate MC-LR into the produced fungal pellets to show uptake ability, which is required for the remediation of toxins from water.

\section{MATERIALS AND METHODS}

\subsection{Microorganism and inoculum preparation}

The fungal strain M. hiemalis EH5 (DSM 14200) was previously isolated as an aquatic $\mathrm{H}_{2} \mathrm{~S}$-resistant strain from the sulfidic-sulfurous Irnsing spring water biofilms in Bavaria, Germany [56]. M. hiemalis cultures were grown on solid malt extract agar substrate as previously reported [44]. Spores were harvested from four-week-old colonies by washing the mycelial surface with sterile distilled water. Suspended sporangiospores were collected in Falcon tubes, centrifuged (5 min, $4000 \times \mathrm{g}$ ) and washed three times with sterile distilled water. The spore concentration was determined using a Neubauer hemocytometer and various concentrations $\left(10^{3}-10^{8}\right.$ spores $\left./ \mathrm{mL}\right)$ were prepared in sterile distilled water for further inoculation procedures described in section 2.3.

\subsection{Culture medium}

Pelletization was tested in three different culture media; (1) nitrogen (N)-limiting medium, adapted from Kirk et al. [57], (2) Sabouraud dextrose broth (SAB) (Sigma-Aldrich) and (3) SAB containing the same vitamin composition as described by Kirk et al. [57]. The vitamin solution consisted of biotin $(5 \mathrm{mg} / \mathrm{L})$, folic acid $(5 \mathrm{mg} / \mathrm{L})$, thiamine hydrochloride $(12.5 \mathrm{mg} / \mathrm{L})$, pyridoxine hydrochloride (12.5 mg/L), cyanocobalamin $(2.5 \mathrm{mg} / \mathrm{L})$, nicotinic acid (12.5 mg/L), DL-calcium pantothenate (12.5 mg/L), $p$ aminobenzoic acid (12.5 mg/L) and thioctic acid (12.5 mg/L) (Sigma-Aldrich) [57]; of which $0.5 \mathrm{~mL}$ was added to one set of $\mathrm{SAB}$ medium per liter. The influence of solid particles was investigated by adding $\mathrm{CaCO}_{3}\left(9 \mathrm{~g} / \mathrm{L}\right.$, Sigma-Aldrich) and $\mathrm{CaO}_{2}$ (0.025\%, Sigma-Aldrich), into the culture medium respectively as described in section 2.3; and trace metals used consisted of $\mathrm{MgSO}_{4}$ - $7 \mathrm{H}_{2} \mathrm{O}$ (25 ppm, Merck), $\mathrm{ZnSO}_{4} \cdot 7 \mathrm{H}_{2} \mathrm{O}$ (4 ppm, Roth), $\mathrm{FeSO}_{4}$ • $7 \mathrm{H}_{2} \mathrm{O}$ (250 ppb, Roth). $\mathrm{HCl}, \mathrm{NaOH}$ and phosphate-buffered saline (PBS, $10 \mathrm{mM}$ ) were used to correct the $\mathrm{pH}$ of the media. PBS was adapted as a $1 \mathrm{x}$ solution following the Cold Spring Harbor Protocols instructions [58].

\subsection{Cultivation method}

Submerged cultivation of $M$. hiemalis was carried out in Erlenmeyer flasks (100 mL medium) on a rotary shaker (Orbital thermoregulated bath 9006, HT INFORS AG) for a maximum of 21 days in the dark. Spore stocks $\left(10^{3}-10^{8}\right.$ spores per milliliter) were prepared by dilution and stored in distilled water at $4{ }^{\circ} \mathrm{C}$ in the dark until inoculation. The inoculation volume was kept constant $(1 \mathrm{~mL})$ resulting in various inoculum sizes (Table 1). Triplicates per test experiment were performed to observe the influence of the cultivation conditions on fungal morphology. Parameters are specified and summarized in Table 1. First, fungal growth and morphology using two different inoculum sizes $\left(10^{2}\right.$ and $10^{4}$ spores $/ \mathrm{mL}$ ) were observed in different media types in order to choose the most appropriate medium for further experiments. Temperature, agitation rate and the addition of calcium carbonate, calcium peroxide and trace metals respectively, were tested in a matrix to observe morphological characteristics. A $\mathrm{pH}$ range from 4 to 8 and inoculum sizes ranging from $10^{1}$ to $10^{6}$ spores $/ \mathrm{mL}$ were tested on the effect of morphological growth. Optimal pH was defined, and the effect on the morphology after adjustment with sodium hydroxide, calcium carbonate and PBS was compared. Normal and baffled shaped flasks, and a change in culture volume (100 and $200 \mathrm{~mL})$ were tested to evaluate the influence on pellet formation.

Table 1: Parameters and ranges tested that influence the probability of pellet formation of $M$. hiemalis in liquid submerged cultivation.

\begin{tabular}{|c|c|c|}
\hline $\begin{array}{c}\text { Parameter } \\
\text { tested }\end{array}$ & Unit & Range \\
\hline Media type & & $\mathrm{N}$-limiting, SAB+V, SAB \\
\hline Temperature & ${ }^{\circ} \mathrm{C}$ & $20,25,30,35,40$ \\
\hline Agitation rate & $\mathrm{rpm}$ & $110,125,130,180$ \\
\hline $\mathrm{pH}$ & & $4,4.5,5,5.5,6,6.5,7,7.5,8$ \\
\hline Inoculum size & $\mathrm{mL}^{-1}$ & $10^{1}, 10^{2}, 10^{3}, 10^{4}, 10^{5}, 10^{6}$ \\
\hline Additives & & $\mathrm{CaCO}_{3}, \mathrm{CaO}_{2}$, trace metals, $\mathrm{HCl}, \mathrm{NaOH}, \mathrm{PBS}$ \\
\hline Flask shape & & normal, baffled \\
\hline Volume & $\mathrm{mL}$ & 100,200 \\
\hline
\end{tabular}

Size variation of pellets was observed by inoculating Erlenmeyer flasks with different amounts of seven-day old pellets $(5,10,20$ and 50 pellets per flask). Flasks containing $100 \mathrm{~mL}$ of SAB medium and the specified amounts of pellets, were incubated at 25 ${ }^{\circ} \mathrm{C}$ and constantly shaken at $130 \mathrm{rpm}$ in the dark. Diameters were measured after 24 and 48 hours of incubation.

\subsection{Analytical methods}

The $\mathrm{pH}$ of the media was measured using a digital $\mathrm{pH}$ meter. The morphological growth form of $M$. hiemalis was observed visually after seven to fourteen days of incubation and was classified into one of four characterized groups: pellets (uniform spherical shaped balls), clumps (non-uniform irregular large aggregates), filaments (freely dispersed mycelia, nonaggregated fungal material), or no growth. Pellet-forming cultures from each flask were dispensed separately in sterile Petri dishes and counted with a sterile pair of tweezers. Biomass was determined as dry weight (dw) after lyophilization $\left(-50.2{ }^{\circ} \mathrm{C}\right.$, 0.1043 mbar for 24 hours in a Lio 5P Freeze-drier, Kambič Laboratorijska oprema d.o.o.).

\subsection{MC-LR extraction method}

For the determination of extraction recovery from the pellets, a defined aliquot of MC-LR (100 ng) was directly added to $50 \mathrm{mg}$ pelletized fungal biomass (dw). Extraction recovery was investigated in triplicate and the established extraction method was 
then applied for toxin extraction from exposed fungi. Lyophilized samples were snap-frozen and disrupted mechanically using a TissueLyzer ( $5 \mathrm{~mm}$ beads, $5 \mathrm{~min}$ treatment, 50/s) until a fine powder was obtained. Then, $0.1 \%$ trifluoroacetic acid in $70 \%$ methanol was added $(5-10 \mu \mathrm{L}$ per $\mathrm{mg} \mathrm{dw})$ and the suspension was sonicated for 60 minutes in an ultrasonic water bath (Allpax, Gmbh \& Co. KG). This was followed by vigorous vortexing for three minutes, followed by centrifugation $(15 \mathrm{~min}, 8000 \mathrm{x} \mathrm{g}$ ). The supernatant was transferred and the remaining pellet re-suspended in half of the volume used at first extraction cycle, repeated twice in total. The remaining pellets were discarded and supernatants were combined and re-centrifuged before analysis on the liquid chromatography tandem mass spectrometer (LC-MS/MS) system. Measurement and quantification of the toxin was performed as previously reported [44].

\subsection{MC-LR exposure}

In order to examine the biosorption ability of pelletized M. hiemalis, seven-day old pellets (with a diameter of $5 \mathrm{~mm}$ ) were transferred into Erlenmeyer flasks containing $50 \mathrm{~mL}$ of $\mathrm{SAB}$ medium. Varying pellet numbers $(3,5,10$ and 25 pellets per flask) were exposed to $100 \mathrm{ng} / \mathrm{mL}$ MC-LR. The control contained three pellets and no toxin to ensure the absence of MC-LR in the fungal strain. The experiment was conducted in triplicate. Sample collection was performed after 48 hours of incubation on a rotary shaker at $130 \mathrm{rpm}$, at $25{ }^{\circ} \mathrm{C}$ in the dark. Pellets were sieved, washed thoroughly with distilled water, snap-frozen and freezedried $\left(-50.2{ }^{\circ} \mathrm{C}, 0.1043\right.$ mbar for 24 hours in a Lio 5P Freeze drier, Kambič Laboratorijska oprema d.o.o.). MC-LR extraction was conducted as described in section 2.5. LC-MS/MS quantification was performed as per Balsano et al. [44].

\subsection{Statistical analysis}

SPSS Statistic software was used for statistical analysis ( $\alpha=0.05,95 \% \mathrm{CI}$ ). Multi-variate analysis was performed as an overall multiple linear regression to investigate the effect of all the factors listed in Table 1 on the morphological form of $M$. hiemalis. Sectional multiple linear regression was additionally applied in order to investigate especially the effect of $\mathrm{pH}$ and inoculum size on the probability of pellet formation. Post-Hoc and t-tests were conducted to analyze uptake and growth results after fungal exposure to MC-LR.

Table 2: Matrix table with factor correlation

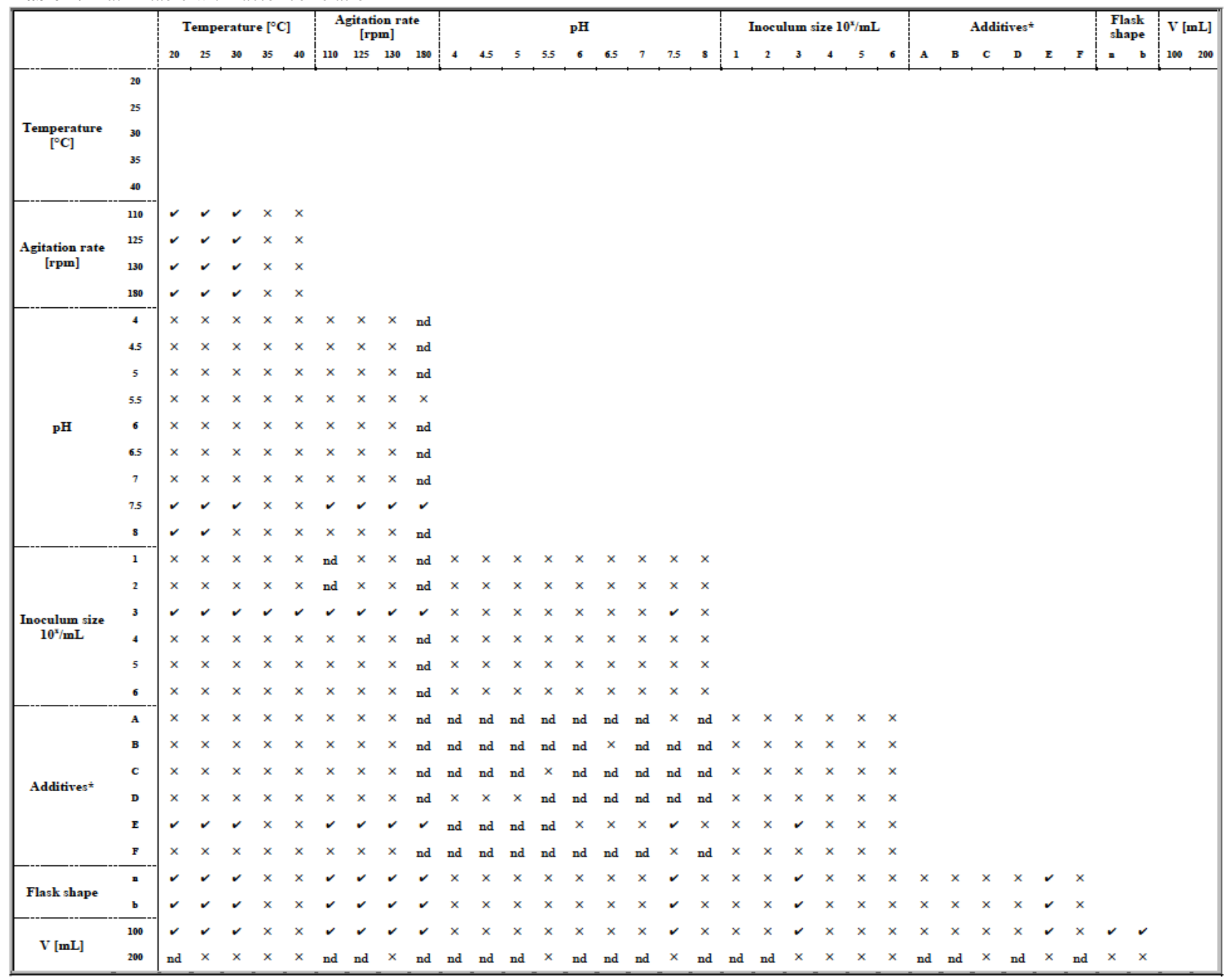

*Additives are $\mathrm{CaCO}_{3}(\mathrm{~A}), \mathrm{CaO}_{2}(\mathrm{~B})$, trace metals (C), $\mathrm{HCl}(\mathrm{D}), \mathrm{NaOH}(\mathrm{E})$, and PBS (F). Flask shape is normal (n) or baffled (b). Tick indicates pellet formation, and cross no pellet formation; nd stands for not determined. 


\section{RESULTS AND DISCUSSION}

Morphological characteristics of filamentous fungi grown in liquid culture strongly depend on the culture parameters. The pelletized form of $M$. hiemalis offers many advantages in industrial purposes and may be useful for application in bioreactor technology for the removal of cyanobacterial toxins from contaminated water. Therefore, different factors that may enhance the probability for pellet formation have been studied. A matrix table was established, which correlates all the tested factors with each other to summarize under which conditions pellet formation of $M$. hiemalis was observed (Table 2). We show a strategic approach by a stepwise optimization of different factors that influence fungal morphology and subsequently analyze the influence of all the factors on the likelihood to promote pellet formation by an overall multivariate linear regression.

The presented overall multiple linear regression model (Equation 1) analyzed the effect of all tested factors on pellet formation (results were characterized as "pellet formation yes/no", Table 2) and predicted that flask shape exhibited significant effects on fungal morphology ( $p=0.011)$, however, medium composition $(p=0.995)$, temperature $(p=0.116)$, agitation rate $(p=0.633)$, inoculum size $(p=0.683)$ and additives $(p=0.993)$ did not have a statistically significant influence on pellet formation if the complete data set was considered. The results on fungal morphology are highlighted in Equation 1.

Morphology $=0.002-(0.002 \times$ media type $)+(0.062 \times$

temperature $)-(0.012 \times$ agitationrate $)+(0.280 \times p H)-$

$(0.051 \times$ inoculum size $)-(0.001 \times$ additives $)+(1.338 \times$

flaskshape $)-(0.011 \times$ volume $)$ Eq. (1)

The equation was developed using SPSS and multiple linear regression. Dependent variable (morphology) and independent variables (factors affecting morphology) were modeled using linear regression analysis and the result was expressed as a linear function of all variables. The unstandardized coefficients calculated by the model have been inserted into a linear function (Equation 1). Accordingly, the low factor values of the additives, media type, volume, and agitation rate, listed in ascending order do not act significantly on fungal morphology. Flask shape and $\mathrm{pH}$ demonstrate strongest effects on fungal morphology, followed by temperature and inoculum size.

\subsection{The influence of media type}

Three different basic types of media were compared to investigate growth behavior and morphological forms of $M$. hiemalis in liquid submerged cultures: The N-limiting variation of the medium developed by Kirk et al. [57], SAB medium with the addition of vitamins $(\mathrm{SAB}+\mathrm{V})$ and $\mathrm{SAB}$ medium without $(\mathrm{SAB})$. Biomass production was characterized as dry weight (lyophilized biomass) after three weeks of cultivation on a continuously shaking rotator. Dry weights were $0.6 \pm 0.03 \mathrm{~g}$ and $0.6 \pm 0.01 \mathrm{~g}$ for $\mathrm{SAB}+\mathrm{V}$ and $\mathrm{SAB}$ respectively, which shows that there is no statistical difference if media were supplemented with vitamins or not $(p=0.991)$. However, growth was found being three fold higher in both SAB media if compared to the fungal growth in the $\mathrm{N}$-limiting medium, where dry weights were only $0.2 \pm 0.03 \mathrm{~g}(p$ $<0.001$ ) (Figure 1).

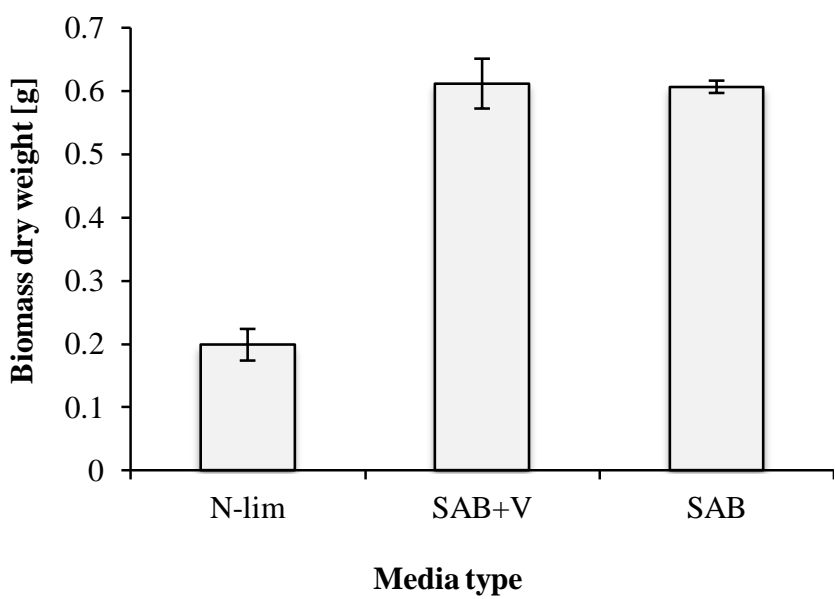

Fig. 1: Biomass production of $M$. hiemalis expressed as dry weights after three weeks of cultivation in different media types (Nitrogen-limiting, Sabouraud dextrose broth with vitamins and Sabouraud dextrose broth without the addition of vitamins) on a rotary shaker. Data represent means \pm standard errors $(n=3)$.

Even though the fungal growth was influenced by the media type, morphology was not. In all three media, with an initial inoculum size of $10^{2}$ sporangiospores per milliliter, the fungus grew in the form of filaments, whereas if the spore concentration was raised to $10^{4}$ spores per milliliter, clumps were prevalent. Opposite results were found for $P$. chrysosporium, where an increased inoculum level led to the transition of clumps to more filamentous morphologies [59] and similar results were found for A. niger, however, much higher inoculum sizes were used in both cases, i.e. $10^{4}$ to $10^{6}, 10^{9}$ spores per milliliter, respectively [52]. However, in this study, media type did only influence the growth extent but not the morphological growth form of $M$. hiemalis. Because of the simpler formulation of the medium, the enhanced growth and the same morphological profile of $M$. hiemalis in SAB without the addition of vitamins, this medium was chosen for further experiments. On the basis of SAB, additional factors were studied to evaluate the effect on fungal morphology. Table 1 shows all the parameters that have been varied to investigate the effect on the morphology of M. hiemalis. Additives $\left(\mathrm{CaCO}_{3}, \mathrm{CaO}_{2}\right.$, and trace metals) that led to the pelletization of other filamentous fungi $[49,55,60,61]$ were tested for the pellet formation of $M$. hiemalis as discussed in section 3.6. $\mathrm{HCl}, \mathrm{NaOH}$ and PBS buffer were used to adjust the $\mathrm{pH}$ of the medium and the effect is discussed in section 3.2.

\subsection{The influence of $\mathbf{p H}$}

The $\mathrm{pH}$ and culture media volume both did not have a statistically significant effect when analyzed by a general multiple regression model, however, $p$-values were lower compared to the other factors ( $p=0.070$ and 0.075 , respectively). Therefore, data was analyzed separately in more detail by sectional multiple linear 
regression in order to elucidate these effects only after optimization of cultural conditions. The effects of the different sources that contributed to an alkaline $\mathrm{pH}$ shift in cultural media were compared. Focusing on the effects caused after addition of sodium hydroxide, the resulting $\mathrm{pH}$ had a statistically significant effect on pellet formation $(p=0.032)$.

The $\mathrm{pH}$ of the culture medium is a measure of the concentration of $\mathrm{H}^{+}$ions present in a solution and contributes to surface phenomena, which might explain its role in relation to pellet formation. The negatively charged functional groups existing on the surface of the fungal cell wall, phosphates, proteins, and carboxylate groups, may undergo protonation at low $\mathrm{pH}$, leading to an increase in the positive charge density on the cell surface. The magnitude of net charge influenced by $\mathrm{pH}$ is species dependent [62].

Here, development of dispersed mycelial filaments of $M$. hiemalis in the $\mathrm{pH}$ range from 4 to 7 were observed, likely due to mutual repulsion. At an acidic to neutral $\mathrm{pH}$, charged cell wall surfaces are predominant in the fungus and hyphae with net charges of the same sign may repel each other and push themselves to grow apart. The exertion of repulsive forces resulted in the growth of dispersed filaments lacking the affinity of fungal cells to aggregate. With the decrease of repulsive electrostatic interactions in correlation with the increase of the $\mathrm{pH}$ toward the cell isoelectrical point, the surface charge decreases, and cells were more likely to aggregate and the fungus grew in the form of intertwined hyphae. Similar observations were reported for $A$. nidulans, where pellet formation was mostly attributed to the $\mathrm{pH}$ dependent electrical charge and hydrophobicity of the conidiospores [53].

Favorable hyphal attraction of $M$. hiemalis was expressed in the range of 7 to 8 , where coagulation of hyphae resulted in the formation of homogenously compact pellets. Within this $\mathrm{pH}$ range after adjustment with sodium hydroxide, filamentous growth did not occur. However, clumpy growth was observed, when $\mathrm{pH}$ reached 7.5 after addition of calcium carbonate or adjustment with phosphate buffer, showing that morphology not only depended on dissociated $\mathrm{H}^{+}$protons but also on the counter ions present in the media, which may interfere with fungal cell surfaces inducing agglomeration. In comparison, $M$. circinelloides formed small pellets with little growth at an initial $\mathrm{pH}$ of 3 . When the $\mathrm{pH}$ was adjusted to 5.3 after 18 hours, cell growth was stimulated, showing compact, spherical, smooth pellets in the presence of $\mathrm{CaCO}_{3}$ and loosely packed, fluffy pellets in the presence of $\mathrm{NaOH}$ [55].

In contrast, M. hiemalis did not pelletize if $\mathrm{pH}$ was lower than 7 and we concluded, that initial $\mathrm{pH}$ adjustment with $\mathrm{NaOH}$ was necessary however, only needed to stimulate pellet formation, and no further $\mathrm{pH}$ control and adjustment was necessary. Once the pellet was formed, the fungus kept growing in the form of a pellet, even if the $\mathrm{pH}$ was not controlled constantly or has dropped due to fungal metabolism. Figure 2 shows the spherical growth of transferred pellets in original SAB medium. This is in contrast with pellet studies on $M$. circinelloides, where $\mathrm{pH}$ was monitored and kept constant at 5.3 in order to enable pellet formation and spherical growth [55].

\subsection{Growth of pellets}

Different amounts of pellets were transferred to new flasks and growth was monitored after 24 and 48 hours of incubation. Pellets kept growing spherically $(p<0.05)$ and reached maximum sizes that were limited by the available space in the flask (Figure 2).
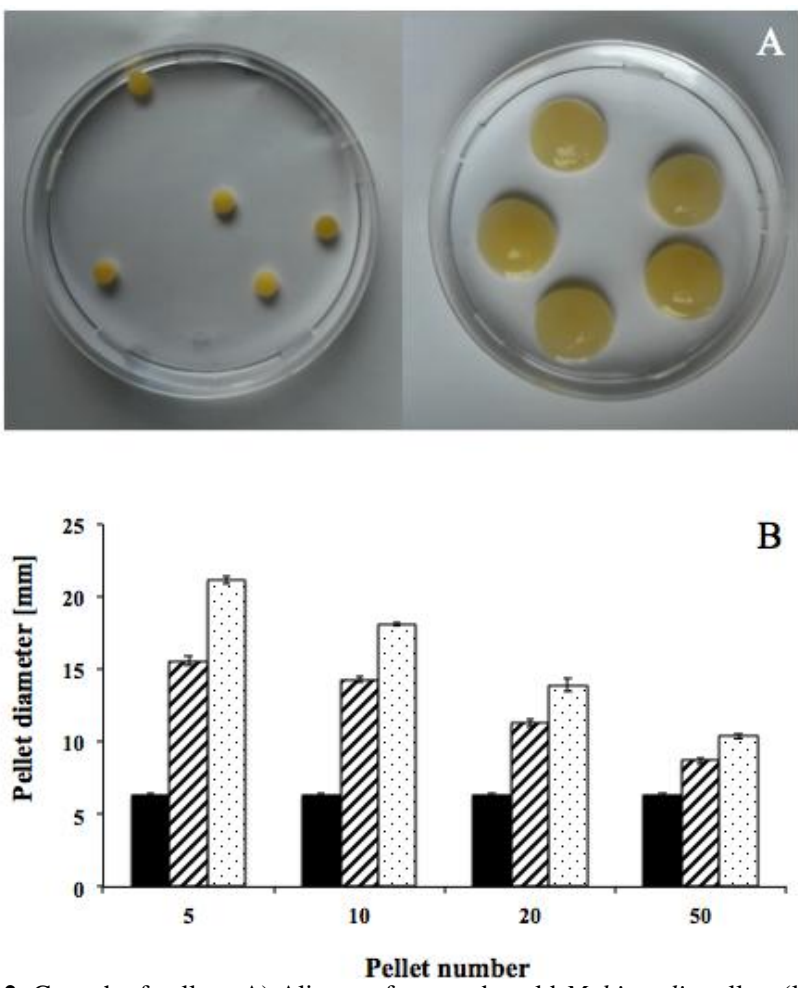

Fig. 2: Growth of pellets. A) Aliquot of seven-day old M. hiemalis pellets (left) and "transferred" pellets after 48 hours, re-incubation in original SAB medium (right). B) Spherical growth of $M$. hiemalis (measured in millimeters) in original SAB medium dependent on the incubation pellet number. Data are means \pm standard errors of five replicates measuring five pellets per replicate. Bars represent measurement points at time point 0,24 and 48 hours (in the order black, striped and dotted).

Figure 2 shows how maximum pellet sizes depended on the inoculation pellet number. Figure $2 \mathrm{~A}$ represents the typical diameter $(5 \mathrm{~mm})$ of the fungal pellets and the larger diameter $(20$ $\mathrm{mm}$ ) achieved when re-inoculated and allowed to grow with more space (Figure 2B). The pellet diameter increased with the decrease in pellet number $(p<0.05)$ monitored after 24 and 48 hours $(p<$ $0.05)$. This shows that sufficient available space is needed for pellets to expand in size and may in part explain why pellet formation was not observed at all spore concentrations within the fixed $\mathrm{pH}$ optimum as explained in section 3.4.

\subsection{The influence of inoculum size}

Separate statistical analysis was used to reveal the influence of inoculum size on fungal growth behavior by means of a sectional multiple linear regression model. All data at concentrations $10^{1}, 10^{2}, 10^{3}, 10^{4}$ spores $/ \mathrm{mL}$ and initial pellet 
inducing $\mathrm{pH}$ control were analyzed in respect to the effect of inoculum size on pellet formation. Our results showed a statistically significant effect of inoculum size on the probability of pellet formation $(p=0.027)$. The optimal spore concentration for pelletization was found to be $10^{3}$ spores per milliliter. Cultures with lower spore concentrations $\left(10^{1}-10^{2}\right.$ spores $\left./ \mathrm{mL}\right)$ resulted in low or not macroscopically visible growth. Cultures with higher spore concentrations $\left(10^{4}-10^{6}\right.$ spores $\left./ \mathrm{mL}\right)$ yielded large clumps. Takahashi and Yamada distinguish two mechanisms of pellet formation, the coagulating and non-coagulating type and it is assumed that the genus Mucor is classified into the noncoagulative type [63]. In this case, spores germinate and young hyphae form small clumps that grow to form pellets. The extreme case of a non-coagulative behavior occurs if inoculum size is small enough that the distance between spores remains large enough to enable each single spore to grow and form one pellet. This explains not only that pellet growth was restricted to space (Figure 2) but also that there must be a critical inoculum size in noncoagulative fungi, which nonetheless depends on the agitation level [64]. Keeping the agitation rate constant at $130 \mathrm{rpm}$ for the cultivation of $M$. hiemalis, biomass was too large if inoculum size was higher than $10^{3}$ spores per milliliter. Exceeding this inoculum size (i.e. $10^{4}-10^{6}$ spores $/ \mathrm{mL}$ ) resulted in clumping of all hyphae and the formation of larger irregular clumps, whereby pellet formation was prevented. At high concentrations the hyphae interact with each other in the early stages of growth, thereby preventing the development of pellets. Therefore, the spore concentration of $10^{3}$ per milliliter has been characterized as the critical inoculum size for $M$. hiemalis, which was two magnitudes higher than for $M$. circinelloides, but much lower in comparison to other pellet-forming fungi. $P$. chrysosporium has been reported to pelletize at a spore concentration of $10^{5} / \mathrm{mL}$ or higher $[65,66]$. The filamentous fungus $R$. oryzae showed pellet formation when $10^{9}$ spores per milliliter were incubated [67]. Inoculum size appeared to be a critical factor in pellet formation; however, $\mathrm{pH}$ changes still provoked the strongest effects on fungal morphology of $M$. hiemalis. Refinement of the initial $\mathrm{pH}$ of the growth medium was crucial for promoting pellet formation, and adjustment of spore concentration was essential in order to provide appropriate space to grow. Interplay of both factors has to be considered for successful pelletization of M. hiemalis.

\subsection{Effect of shear forces}

Additionally, in the present study factors connected to impact on mechanical forces have been investigated. Among them, volume had substantial effects on pellet formation. Pellets were only formed when $100 \mathrm{~mL}$ media were used, whereas in $200 \mathrm{~mL}$ no pellet formation was observed. A change in volume creates a different force and resulting fluid dynamics. It is possible that a larger volume requires higher agitation speed to enable a comparable liquid movement required for pelletization. However, this was not tested here. Moreover, too much volume reduces oxygen transfer, whereby a smaller volume maximizes the surface area exposed to the available air and produces sufficient supply of oxygen and removal of carbon dioxide [68]. Flask shape had negligible effects on the morphology under optimized medium composition conditions, however smoother pellets were obtained if baffled flasks were used rather than normal shaped flasks, showing that additional shear forces slightly influenced pellet characteristics. Agitation was important for good mixing to keep spore-to-spore distance large enough to prevent clumpy growth, and moreover it created shear forces, which influenced fungal morphology. The agitation rate (110-180 rpm), however, did not have a statistical significant role in pellet formation $(p=0.633)$.

\subsection{Effect of additives}

The supplementation of additives $\left(\mathrm{CaCO}_{3}, \mathrm{CaO}_{2}\right.$, or trace metals) did not exhibit positive effects on pellet formation in contrast to what was reported for other filamentous fungi [49, 55, $60,61]$. Trace metals in the cultivation medium significantly promoted the formation of pellets of $R$. oryzae ATCC 20344 [49, 60], whereas in the present study trace element addition did not promote pelletization. Addition of solid particles, such as calcium carbonate, which was barely soluble in the medium, showed a clear negative effect on the probability of pelletization of $M$. hiemalis. Conversely, numerous studies showed that the addition of solid particles enhanced the probability of pelletization of different fungi, e.g. the addition of rice promoted pellet formation of $R$. oryzae [67] and titanate ( $\left.\mathrm{TiSiO}_{4}\right)$ microparticles resulted in the pelletization of A. niger [69]. Moreover, the addition of calcium carbonate not only favored pellet formation in $R$. oryzae [70] but also induced pelletization of the species circinelloides of the genus Mucor [55]. Generally, the addition of calcium ions (in the form of $\mathrm{CaCO}_{3}$ or $\mathrm{CaO}_{2}$ ) both did not promote pellet formation of $M$. hiemalis, however in $R$. oryzae and A. niger, the addition of $\mathrm{Ca}^{2+}$ ions in the form of $\mathrm{CaCl}_{2}$ resulted in the production of pellets for both fungi [50,71].

\subsection{Effect of temperature}

In regards to cultural temperature, M. hiemalis grew from $23{ }^{\circ} \mathrm{C}$ to $30^{\circ} \mathrm{C}$. Variation of the temperature within that range did not have an influence on pellet probability, but fungal growth was inhibited if the cultivation temperature reached $40{ }^{\circ} \mathrm{C}$. Future studies are required to investigate growth and biosorption potential of pelletized M. hiemalis at lower temperatures.

By modifying the cultivation conditions, the filamentous fungus $M$. hiemalis was successfully manipulated into growing as pellets. In summary, a total of eight different factors and their synergistic effects were considered. The factors included media type, temperature, agitation rate, inoculum size, $\mathrm{pH}$, additives, flask shape, and volume. The different combinations resulted in growth, ranging from large irregular shaped clumps or spherical pellets to completely dispersed mycelia. When comparing morphological results to other fungi tested in literature, it clearly shows that influence not only varies from genus to genus, but also between species. A multifactorial experimental design developed and applied to Rhizopus sp. showed that temperature not only led to a faster development but also increased the probability, and 
agitation rate, calcium ion concentration, $\mathrm{pH}$ and solid cellulose particles each had significant effects on pelletization of the fungus, whereas inoculum size and liquid volume, both factors that showed to influence $M$. hiemalis pellet formation, were not found to have a significant effect on Rhizopus sp. [50]. While $\mathrm{pH}$ adjustment during cell growth via addition of calcium carbonate was used to induce pellet formation of M.circinelloides [55], the addition of calcium carbonate suppressed pellet formation of the Mucor species in question and constant $\mathrm{pH}$ control was not necessary for pellet growth. Obviously, the factors influencing fungal morphology are many that have to be considered, and only an experimental adaptation of the culture parameters allows pellet formation for the fungus in question.

\subsection{MC-LR uptake by fungal pellets of $M$. hiemalis}

Uptake experiments were performed to examine the biosorption capacity of M. hiemalis at varying pellet numbers per exposure flask. The extraction method used to isolate MC-LR from fungal pellets yielded excellent recoveries $(100 \pm 2 \%)$ as a total of $97.8 \pm 2.5 \mathrm{ng}$ MC-LR $(n=3)$ were quantified via LCMS/MS analysis in the test samples containing $50 \mathrm{mg}$ untreated pelleted fungal biomass (dw) and $100 \mathrm{ng}$ MC-LR. The same extraction procedure was applied to toxin treated samples and extracted toxin was analyzed and quantified via LC-MS/MS. The fungus showed uptake between 0.2-0.4 micrograms MC-LR per gram pelletized biomass $(\mathrm{dw})(p<0.05)$ for all pellet numbers tested. No statistical difference of uptake could be observed when comparing 3,5 or 10 pellets $(p>0.05)$. However, a statistically higher concentration of MC-LR was detected when 25 pellets per flasks were used $(p<0.05)$. Figure 3 graphically shows the total uptake in nanograms that was achieved by using pelletized $M$. hiemalis. Growth expressed as milligram dry weight after 48 hours of exposure was plotted on a secondary axis. The ratio between total uptake and biomass lies in the same range for all the pellet numbers $(p>0.05)$, which explains why a statistically higher amount of toxin has been biosorbed within the pellets when 25 pellets were used, as a much higher amount of biomass was obtained after 48 hours of incubation and exposure $(p<0.05)$. Comparing biomass of three pellets exposed to the toxin to three pellets of untreated controls, we observed that no statistically significant growth variation occurred $(p=0.365)$. This explains that growth behavior of the fungus was not negatively affected in the presence of cyanotoxin, which confirms the results obtained in a previous study [44].

To the authors' knowledge, this is the first report to introduce cyanobacterial toxin uptake in pelletized filamentous fungi. Further studies are suggested to enhance biosorption ability using larger fungal biomass and a longer exposure range, as well as examine the optimal concentration range for best toxin removal. It can be concluded that $M$. hiemalis is an attractive organism to be further studied for the capacity expansion of the approved Green Liver Systems ${ }^{\circledR}$ due to the similar biosorption potential of the fungus towards MC-LR compared to several aquatic plants. In $M$. hiemalis pellets, $0.7 \%$ of the total applied MC-LR was internalized when using 25 pellets. This is similar compared to the uptake reported in Ceratophyllum demersum, Elodea canadensis and Vesicularia dubyana, where $0.6-1.75 \%$ of the total toxin exposure concentration could be detected intracellularly [72]. Lemna minor and Chladophora fracta were exposed to a concentration that was 100-200 times higher than what was applied to M. hiemalis cultures in the present study, nonetheless, similar intracellular toxin concentrations were detected and this only after a prolonged exposure of five days [73]. The present study focuses only on fungal uptake of MC-LR, but further studies are suggested to investigate possible degradation of the toxin via extracellular or intracellular enzymatic pathways in the fungus. Toxin removal from the water could be achieved by using $M$. hiemalis pellets in bioreactor technology, which inspires future work on pelletized $M$. hiemalis as a mycoremediation tool in bioreactors for the removal of cyanobacterial as well as other hazardous toxins from contaminated water.

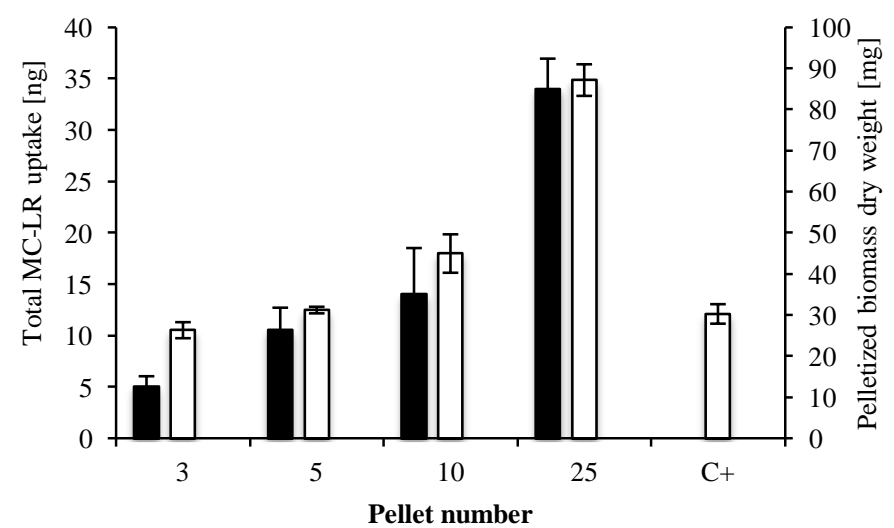

Fig. 3: Microcystin-LR uptake in fungal pellets of $M$. hiemalis. Total toxin uptake $[n g]$ plotted on the primary axis (black bars) and fungal biomass expressed as lyophilized dry weight [mg] plotted on the secondary axis (white bars) after 48 hours of incubation and exposure to $100 \mathrm{ng} / \mathrm{mL}$ of the cyanotoxin versus different amounts of fungal pellets. The positive control $(\mathrm{C}+)$ in the absence of toxin contained three pellets. Data represent means \pm standard errors $(n=3)$.

\section{CONCLUSIONS}

The present study is the first report of a cultivation method for the pellet production of $M$. hiemalis in liquid submerged cultures. When comparing factors that influence fungal morphology, there is a clear difference not only between genera, but also between species of the same genus. Initial sodium hydroxide based $\mathrm{pH}$ adjustment of Sabouraud dextrose broth medium was crucial for the pellet induction in M. hiemalis cultures with inoculum size of $10^{3}$ spores per milliliter in baffled (or normal shaped) Erlenmeyer flasks with a volume of $100 \mathrm{~mL}$ medium. The study combines the application of the obtained fungal pellets with biosorption experiments towards the cyanotoxin microcystin-LR. Toxin uptake into fungal pellets was demonstrated, which is an essential prerequisite to the applicability of an organism in water remediation. The results motivate further work in order to establish fungal bioreactors that may be used for efficient cyanobacterial as well as other toxin removal from contaminated water. 


\section{ACKNOWLEDGEMENT}

We gratefully acknowledge the state of Berlin for providing the Elsa-Neumann-Scholarship to Evelyn Balsano in the context of Nachwuchsförderungsgesetz-NaFöG. The authors thank Dr. V. Contardo-Jara for scientific input and S. Kühn for assistance with LC-MS/MS measurements.

\section{REFERENCES}

1. Imanishi S, Kato H, Mizuno M, Tsuji K, Harada KI. Bacterial degradation of microcystins and nodularin. Chemical Research in Toxicology. 2005; 18(3):591-598.

2. Namikoshi M, Sivonen K, Evans WR, Sun F, Carmichael WW, Rinehart KL. Isolation and structures of microcystins from a cyanobacterial water bloom (Finland). Toxicon. 1992; 30(11):14731479.

3. Luukkainen R, Namikoshi M, Sivonen K, Rinehart KL, Niemelä SI. Isolation and identification of 12 microcystins from four strains and two bloom samples of Microcystis new hepatotoxin. Toxicon. 1994; 32(1):133-139.

4. Sivonen K, Namikoshi M, Evans WR, Färdig M, Carmichael WW, Rinehart KL. Three new microcystins, cyclic heptapeptide hepatotoxins, from Nostoc sp. strain 152. Chemical Research in Toxicology. 1992; 5(4):464-469.

5. Lanaras T, Cook CM. Toxin extraction from an Anabaenopsis milleri - dominated bloom. Science of The Total Environment. 1994; 142(3):163-169.

6. Azevedo SMFO, Carmichael WW, Jochimsen EM, Rinehart KL, Lau S, Shaw GR, Eaglesham GK. Human intoxication by microcystins during renal dialysis treatment in Caruaru-Brazil. Toxicology. 2002; 181-182(0):441-446.

7. Jochimsen EM, Carmichael WW, An J, Cardo DM, Cookson ST, Holmes CEM, Antunes MB, de Melo Filho DA, Lyra TM, Barreto VST, Azevedo SMFO, Jarvis WR. Liver failure and death after exposure to microcystins at a hemodialysis center in Brazil. New England Journal of Medicine. 1998; 338(13):873-878.

8. Falconer IR, Humpage AR. Tumour promotion by cyanobacterial toxins. Phycologia. 1996; 35(6S):74-79.

9. Falconer IR. Tumor promotion and liver injury caused by oral consumption of cyanobacteria. Environmental Toxicology and Water Quality: An International Journal. 1991; 6(2):177-184.

10. Nishiwaki-Matsushima $R$, Ohta $T$, Nishiwaki $S$, Suganuma $M$, Kohyama K, Ishikawa T, Carmichael WW, Fujiki H. Liver tumor promotion by the cyanobacterial cyclic peptide toxin microcystin-LR. Journal of Cancer Research and Clinical Oncology. 1992; 118(6):420-424.

11. Yoshida T, Makita Y, Nagata S, Tsutsumi T, Yoshida F, Sekijma M, Tamura S, Ueno Y. Acute oral toxicity of microcystin-LR, a cyanobacterial hepatotoxin, in mice. Natural Toxins. 1997; 5(3): 9195.

12. DeMott WR, Zhang QX, Carmichael WW. Effects of toxic cyanobacteria and purified toxins on the survival and feeding of a copepod and three species of Daphnia. Limnology and Oceanography. 1991; 36(7):1346-1357.

13. Ghazali IE, Saqrane S, Carvalho AP, Ouahid Y, Oudra B, del Campo $\mathrm{FF}$, Vasconcelos V. Compensatory growth induced in zebrafish larvae after pre-exposure to a Microcystis aeruginosa natural bloom extract containing microcystins. International Journal of Molecular Sciences. 2009; 10(1):133-146.

14. Saqrane S, Ghazali IE, Ouahid Y, Hassni ME, Hadrami IE, Bouarab L, del Campo FF, Oudra B, Vasconcelos V. Phytotoxic effects of cyanobacteria extract on the aquatic plant Lemna gibba: Microcystin accumulation, detoxication and oxidative stress induction. Aquatic Toxicology. 2007; 83(4):284-294.

15. LeBlanc S, Pick FR, Aranda-Rodriguez R. Allelopathic effects of the toxic cyanobacterium Microcystis aeruginosa on duckweed, Lemna gibba L. Environmental Toxicology. 2005; 20(1):67-73.
16. Pflugmacher S, Codd GA, Steinberg CEW. Effects of the cyanobacterial toxin microcystin-LR on detoxication enzymes inaquatic plants. Environmental Toxicology. 1999; 14(1):111-115.

17. Pflugmacher S. Possible allelopathic effects of cyanotoxins, with reference to microcystin-LR, in aquatic ecosystems. Environmental Toxicology. 2002; 17(4):407-413.

18. Kormas KA, Lymperopoulou DS. Cyanobacterial toxin degrading bacteria: Who are they? BioMed Research International. 2013; 2013(0):1-12.

19. Pflugmacher S, Kühn S, Lee SH, Choi JW, Baik S, Kwon KS, Contardo-Jara V. Green liver systems ${ }^{\circledR}$ for water purification - using the phytoremediation potential of aquatic macrophytes for the removal of different cyanobacterial toxins from water. American Journal of Plant Sciences. 2015; 6(9):1607-1618.

20. Nimptsch J, Wiegand C, Pflugmacher S. Cyanobacterial toxin elimination via bioaccumulation of MC-LR in aquatic macrophytes: An application of the "Green Liver Concept". Environmental Science \& Technology. 2008; 42(22):8552-8557.

21. Tien M. Properties of ligninase from Phanerochaete chrysosporium and their possible applications. Critical Reviews in Microbiology. 1987; 15(2):141-168.

22. Ulmer D, Leisola M, Puhakka J, Fiechter A. Phanerochaete chrysosporium: Growth pattern and lignin degradation. European Journal of Applied Microbiology and Biotechnology. 1983; 18(3):153-157.

23. Novotný Č, Svobodová K, Erbanová P, Cajthaml T, Kasinath A, Lang E, Šašek V. Ligninolytic fungi in bioremediation: Extracellular enzyme production and degradation rate. Soil Biology and Biochemistry. 2004; 36(10):1545-1551.

24. Reddy CA. The potential for white-rot fungi in the treatment of pollutants. Current Opinion in Biotechnology. 1995; 6(3):320-328.

25. Arjmand M, Sandermann Jr H. N-(Chlorophenyl)-succinimides: A novel metabolite class isolated from Phanerochaete chrysosporium. Pesticide Biochemistry and Physiology. 1987; 27(2):173-181.

26. Arjmand M, Sandermann Jr H. Mineralization of chloraniline/lignin conjugates and of free chloranilines by the white-rot fungus Phanerochaete chrysosporium. Journal of Agricultural and Food Chemistry. 1985; 33(6):1055-1060.

27. Hickey WJ, Fuster DJ, Lamar RT. Transformation of atrazine in soil by Phanerochaete chrysosporium. Soil Biology and Biochemistry. 1994; 26(12):1665-1671.

28. Mougin C, Laugero C, Asther M, Dubroca J, Frasse P, Asther M. Biotransformation of the herbicide atrazine by the white-rot fungus Phanerochaete chrysosporium. Applied and Environmental Microbiology. 1994; 60(2):705-708.

29. Bumpus JA. Biodegradation of polycyclic aromatic hydrocarbons by Phanerochaete chrysosporium. Applied and Environmental Microbiology. 1989; 55(1):154-158.

30. Eaton DC. Mineralization of polychlorinated biphenyls by Phanerochaete chrysosporium: A ligninolytic fungus. Enzyme and Microbial Technology. 1985; 7(5):194-196.

31. Fernando T, Bumpus JA, Aust SD. Biodegradation of TNT $(2,4,6$ trinitrotoluene) by Phanerochaete chrysosporium. Applied and Environmental Microbiology. 1990; 56(6):1666-1671.

32. Mileski G, Bumpus JA, JurekMA, Aust SD. Biodegradation of pentachlorophenol by the white-rot fungus Phanerochaete chrysosporium. Applied and Environmental Microbiology. 1988; 54(12):2885-2889.

33. Joshi DK, Gold MH. Degradation of 2,4,5-trichlorophenol by the lignin-degrading basidiomycete Phanerochaete chrysosporium. Applied and Environmental Microbiology. 1993; 59(6):1779-1785.

34. Cammarota MC, Sant'Anna Jr GL. Decolorization of kraft bleach plant $E_{1}$ stage effluent in a fungal bioreactor. Environmental Technology. 1992; 13(1):65-71.

35. Lu Y, Yan L, Wang Y, Zhou S, Fu J, Zhang J. Biodegradation of phenolic compounds from coking wastewater by immobilized white rot fungus Phanerochaete chrysosporium. Journal of Hazardous Materials. 2009; 165(1-3):1091-1097. 
36. Miranda Rde C, Gomes Ede B, Pereira Jr N, Marin-Morales MA, Machado KM, Gusmão NB. Biotreatment of textile effluent in static bioreactor by Curvularia lunata URM 6179 and Phanerochaete chrysosporium URM 6181. Bioresource Technology. 2013; 142(0):361-367.

37. Mielgo I, Moreira MT, Feijoo G, Lema JM. Biodegradation of a polymeric dye in a pulsed bed bioreactor by immobilized Phanerochaete chrysosporium. Water Research. 2002; 36(7):18961901.

38. Lamar RT, Larsen MJ, Kirk TK, Glaser JA. Growth of the white-rot fungus Phanerochaete chrysosporium in soil. In: Barkley NP, Martin $\mathrm{JF}$, editors. Land disposal, remedial action, incineration and treatment of hazardous waste: Proceedings of the 13th annual research symposium, U.S. EPA, Cincinnati, OH: Hazardous waste and engineering research laboratory, Office of research and development; 1987, p. 419-424.

39. Hoque E, inventor; Patent De, assignee. Verfahren zum Abbau von Xenobiotika durch Pilzarten mit Monooxygenase-/ DioxygenaseAktivität in Gegenwart von Pilzen mit Glutathion- $S$-TransferaseAktivität. Deutsches Patentdokument DE10125365C2. 2003 June 5.

40. Rønhede S, Jensen B, Rosendahl S, Kragelund BB, Juhler RK, Aamand J. Hydroxylation of the herbicide isoproturon by fungi isolated from agricultural soil. Applied and Environmental Microbiology. 2005; 71(12):7927-7932.

41. Shroff KA, Vaidya VK. Kinetics and equilibrium studies on biosorption of nickel from aqueous solution by dead fungal biomass of Mucor hiemalis. Chemical Engineering Journal. 2011; 171(3):1234-1245.

42. Tewari N, Vasudevan P, Guha BK. Study on biosorption of $\mathrm{Cr}(\mathrm{VI})$ by Mucor hiemalis. Biochemical Engineering Journal. 2005; 23(2):185-192.

43. Fritscher J, Hoque E, Stöckl M, inventor; PatentDe, assignee. Fungus Mucor hiemalis, useful for the removal of heavy metals e.g. mercury, chromium, uranium and aluminum in ground- and surface water, purification plants, waste water and industrial water is new. Deutsches Patentdokument DE102004020837B4. 2006 Feb 23.

44. Balsano E, Esterhuizen-Londt M, Hoque E, Pflugmacher S. Toxin resistance in aquatic fungi poses environmentally friendly remediation possibilities: A study on the growth responses and biosorption potential of Mucor hiemalis EH5 against cyanobacterial toxins. International Journal of Water and Wastewater Treatment. 2015; 1(1):1-9.

45. Gibbs PA, Seviour RJ, Schmid F. Growth of filamentous fungi in submerged culture: Problems and possible solutions. Critical Reviews in Biotechnology. 2000; 20(1):17-48.

46. Kim JH, Lebeault JM, Reuss M. Comparative study on rheological properties of mycelial broth in filamentous and pelleted forms. European Journal of Applied Microbiology and Biotechnology. 1983; 18(1):11-16.

47. Olsvik E, Kristiansen B. Rheology of filamentous fermentations. Biotechnology Advances. 1994; 12(1):1-39.

48. Liao W, Liu Y, Chen S. Studying pellet formation of a filamentous fungus Rhizopus oryzae to enhance organic acid production. In: Mielenz JR, Klasson KT, Adney WS, McMillan JD, editors. Applied Biochemistry and Biotechnology, Biotechnology for Fuels and Chemicals, The Twenty-Eight Symposium, New York: Humana Press; 2007, p. 689-701.

49. Liao W, Liu Y, Frear C, Chen S. A new approach of pellet formation of a filamentous fungus - Rhizopus oryzae. Bioresource Technology. 2007; 98(18):3415-3423.

50. Nyman J, Lacintra MG, Westman JO, Berglin M, Lundin M, Lennartsson PR, Taherzadeh MJ. Pellet formation of zygomycetes and immobilization of yeast. New Biotechnology. 2013; 30(5):516522 .

51. Žnidaršič P, Komel R, Pavko A. Studies of a pelleted growth form of Rhizopus nigricans as a biocatalyst for progesterone $11 \alpha$ hydroxylation. Journal of Biotechnology. 1998; 60(3):207-216.

52. Papagianni M, Mattey M. Morphological development of Aspergillus niger in submerged citric acid fermentation as a function of the spore inoculum level. Application of neural network and cluster analysis for characterization of mycelial morphology. Microbial Cell Factories. 2006; 5(3):1-12.

53. Dynesen J, Nielsen J. Surface hydrophobicity of Aspergillus nidulans conidiospores and its role in pellet formation. Biotechnology Progress. 2003; 19(3):1049-1052.

54. Carlsen M, Spohr AB, Nielsen J, Villadsen J. Morphology and physiology of an $\alpha$-amylase producing strain of Aspergillus oryzae during batch cultivations. Biotechnology and Bioengineering. 1996; 49(3):266-276.

55. Xia C, Zhang J, Zhang W, Hu B. A new cultivation method for microbial oil production: Cell pelletization and lipid accumulation by Mucor circinelloides. Biotechnology for Biofuels. 2011; 4(15):1-10.

56. Hoque E, Pflugmacher S, Fritscher J, Wolf $M$. Induction of glutathione $S$-transferase in biofilms and germinating spores of Mucor hiemalisstrain EH5 from cold sulfidic spring waters. Applied and Environmental Microbiology. 2007; 73(8):2697-2707.

57. Kirk TK, Schultz E, Connors WJ, Lorenz LF, Zeikus JG. Influence of culture parameters on lignin metabolism by Phanerochaete chrysosporium. Archives of Microbiology. 1978; 117(3):277-285.

58. Sambrook J, Russel T. Preparation of reagents and buffers used in molecular cloning, phosphate-buffered saline (PBS). In: Argentine J, Irwin N, Janssen KA, Curtis S, Zierler M, McInerny N, Brown D, Schaefer S, editors. Molecular cloning: a laboratory manual, New York: Cold Spring Harbor Laboratory Press; 2001, p. A1.7.

59. Tucker KG, Thomas CR. Mycelial morphology: The effect of spore inoculum level. Biotechnology Letters. 1992; 14(11):1071-1074.

60. Zhou Y, Du J, Tsao GT. Mycelial pellet formation by Rhizopus oryzae ATCC 20344. Applied Biochemistry and Biotechnology. 2000; 84(1):779-789.

61. Sladdin M, Lynch JM. Antimicrobial properties of calcium peroxide in relation to its potential use as a seed dressing. Journal of General Microbiology. 1983; 129(7):2307-2314.

62. Douglas HW, Collins AE, Parkinson D. Electric charge and other surface properties of some fungal spores. Biochimica et Biophysica Acta. 1959; 33(2):535-538.

63. Takahashi J, Yamada H. Studies on the effect of some physical conditions on the submerged mold culture. Part II. On the two types of pellet formation in the shaking culture. Journal of Agricultural Chemical Society. 1959; 33(8):707-710.

64. Van Suijdam JC, Kossen NWF, Paul PG. An inoculum technique for the production of fungal pellets. European Journal of Applied Microbiology and Biotechnology. 1980; 10(3):211-221.

65. Gerin PA, Dufrene Y, Bellon-Fontaine MN, Asther M, Rouxhet PG. Surface properties of the conidiospores of Phanerochaete chrysosporium and their relevance to pellet formation. Journal of Bacteriology. 1993; 175(16):5135-5144.

66. Wu J, Li QB. Biosorption of lead by Phanerochaete chrysosporium in the form of pellets. Journal of Environmental Sciences. 2002; 14(1):108-114

67. Liu Y, Liao W, Chen S. Study of pellet formation of filamentous fungi Rhizopus oryzae using a multiple logistic regression model Biotechnology and Bioengineering. 2008; 99(1):117-128.

68. Henzler HJ, Schedel M. Suitability of the shaking flask for oxygen supply to microbiological cultures. Bioprocess Engineering. 1991; 7(3):123-131.

69. Driouch H, Hänsch R, Wucherpfennig T, Krull R, Wittmann C. Improved enzyme production by bio-pellets of Aspergillus niger: Targeted morphology engineering using titanate microparticles. Biotechnology and Bioengineering. 2012; 109(2):462-471.

70. Liu Y, Liao W, Chen S. Co-production of lactic acid and chitin using a pelletized filamentous fungus Rhizopus oryzae cultured on cull potatoes and glucose. Journal of Applied Microbiology. 2008; 105(5):1521-1528.

71. Pera LM, Callieri DA. Influence of calcium on fungal growth, hyphal morphology and citric acid production in Aspergillus niger. Folia Microbiologica. 1997; 42(6):551-556. 
72. Pflugmacher S, Wiegand C, Beattie KA, Codd GA, Steinberg C. Uptake of the cyanobacterial hepatotoxin microcystin-LR by aquatic macrophytes. Journal of Applied Botany. 1998; 72(5-6):228-232.

73. Mitrovic SM, Allis O, Furey A, James KJ. Bioaccumulation and harmful effects of microcystin-LR in the aquatic plants Lemna minor and Wolffia arrhiza and the filamentous alga Chladophora fracta. Ecotoxicology and Environmental Safety. 2005; 61(3):345-352.

\section{How to cite this article:}

Balsano E, Esterhuizen-Londt M, Hoque E, Pflugmacher S. Fungal pellets as potential tools to control water pollution: Strategic approach for the pelletization and subsequent microcystin-LR uptake by Mucor hiemalis. J App Biol Biotech. 2016; 4 (04): 031-041. DOI: 10.7324/JABB.2016.40403 